\title{
Can Growing Popular Support for Physician-Assisted Death Motivate Organized Medicine to Improve End-of-Life Care?
}

\author{
Elizabeth Dzeng, $M D, P h D, M P H^{1,2}$ \\ 'Department of Medicine, Division of Palliative Medicine, University of California, San Francisco, San Francisco, CA, USA; ${ }^{2}$ Department of Social and \\ Behavioral Science, Sociology Program, University of California, San Francisco, San Francisco, CA, USA.
}

J Gen Intern Med 33(8):1209-11

DOI: $10.1007 / \mathrm{s} 11606-018-4485-8$

(c) Society of General Internal Medicine 2018

$\mathrm{M}$ odern medicine has experienced tectonic shifts over the last several decades from a technological, ethical, and sociological perspective, particularly around care at the end of life. The confluence of these transformations has shaped the public discourse around the way we die and has given rise to the debates we see today surrounding physician-assisted death (PAD). Through personal stories and stories in the popular press regarding overly aggressive treatments at the end of life, some Americans are fearful that they too might die what is often portrayed as a dehumanizing death. The increasing momentum towards legalization of PAD is in part a reaction to this public perception embedded in a clinical reality and motivated by a desire to regain personal control. This, it can be assumed, would counteract a perceived helplessness to resist the overwhelming array of choices within a system that defaults to aggressive care at the end of life.

In this issue of the Journal of General Internal Medicine, Sulmasy and colleagues lay out an argument for why neutrality on PAD by professional medical organizations is neither appropriate nor neutral ${ }^{1}$. They stress that neither the fact that the membership disagree on the moral acceptability of PAD nor that physicians in seven states (most recently Hawaii) and the District of Columbia are permitted to prescribe the lethal drug requires a position of neutrality. They argue that neutrality is not neutral as it "declares a policy no longer morally unacceptable." The authors base their arguments against PAD on the "meaning of medical practice, the importance of the physician-patient relationship, and respect for the common good." They contend that autonomy alone does not justify PAD, as it must be weighed by the other ethical principles such as justice, beneficence (best interest), and non-maleficence (first do no harm). They also argue that PAD is not a healing act and can also cause further harm by jeopardizing the trust inherent in the physician-patient relationship.

Sulmasy et al. highlight PAD's socio-political implications and the effects of legalization on public opinion of PAD. They argue that allowing PAD can send social

Published online May 18, 2018 messages, including one that devalues lives by suggesting that there are certain states that are not worth living. Legalizing PAD could slowly change social norms that might encourage suicide contagion, normalize PAD leading to an incremental extension of PAD, and further perpetuate the image of pain and suffering as an inevitable part of death.

The focus on the implications of PAD on normative thinking and public opinion is important and speaks to the social complexity of this issue. While some might argue that PAD is appropriate for certain individuals (i.e., a well-informed, empowered patient in a trusting relationship with a physician who is willing to prescribe), one cannot ignore the aggregate effects of individual actions on broader social perceptions. This I believe is where the greatest danger lies in the legalization of PAD. There are additional social and historical forces that might explain why PAD has risen to the forefront of public discourse today. By exploring the root causes of the movement to legalize PAD, we can better understand and address the problems inherent in end-of-life care that contributed to this groundswell of support for PAD.

Life-sustaining technologies such as mechanical ventilation and extracorporeal membranous oxygenation (ECMO) as well as powerful new chemotherapy and biologic agents mean that attempting to delay death at all costs is becoming an increasingly viable option. Sometimes forgotten in this quest is the permission to prioritize quality of life over longevity. A default of aggressive care in the hospital, financial incentives to overtreat, social pressures to be a "fighter," and the inherent emotional trauma and fears of death, further encourage a culture of care that prioritizes high-intensity care at the expense of humanistic care.

As a result, despite efforts to improve palliative care and advance care planning, intensity of end-of-life care continues to increase in America. For example, rates of ICU admissions and life-sustaining treatments continue to rise among older adults ${ }^{2}$ despite evidence suggesting that life-sustaining therapies in the ICU may not always be beneficial and might even be at times harmful. ${ }^{3,4}$ Overly aggressive treatments further cause harm by reducing quality of life near death and exacerbating caregiver bereavement. ${ }^{5,6}$ When burdensome end-oflife care occurs, it is difficult to maintain that non-maleficence has been honored and that medicine is fulfilling its fundamental calling to provide person-centered, empathetic care. 
The challenges and suffering inherent in a treatment-at-allcosts approach has been increasingly highlighted in the popular press through writing by Atul Gawande ${ }^{7}$, Katy Butler ${ }^{8}$, and others. It should thus not be a surprising consequence that the public increasingly feels a need to empower themselves against prolonged suffering caused by this culture of overly aggressive care. The increasing acceptance of PAD I suspect, in part lies with an insufficient emphasis on non-maleficence in the drive to sustain life in terminal conditions when treatments become burdensome. The argument that PAD is morally unacceptable because physicians have sworn an oath to do no harm and to first and foremost, heal, becomes less compelling.

These challenges occur on a backdrop of an American cultural philosophy that prioritizes individual rights, autonomy, and the primacy of control over one's own destiny. While America was founded on the tenet of rugged individualism ${ }^{9}$, significant shifts in the physician-patient relationship from paternalism to autonomy have only occurred over the past several decades. At the core of this transformation was the broader movement towards the neoliberal values of individualism and the supremacy of competitive market forces in the 1980s. This laid the foundation for a shift from what Talcott Parsons (1950) described as the "sick role"10 where patients faithfully followed their doctor's instructions in order to get better, towards one that emphasized an autonomous patient consumer who could choose doctors and treatments based on their personal preferences.

While a shift away from physician paternalism was necessary, some worry that the pendulum has swung too far, and clinicians risk abandoning patients to make their own decisions. In a qualitative interview study of physicians, I found that hospital cultures that prioritized autonomy encouraged physician trainees to withhold recommendations because they feared that it would hinder patient autonomy ${ }^{11}$. Rather than empowering patients, a dogmatic ideology of autonomy and choice instead withdraws physicians from their responsibility to guide and abandons patients at their most vulnerable moment. I believe this lack of guidance contributes both to the trends towards overly aggressive end-of-life care and to the desire to exercise PAD to mitigate helplessness when faced with overwhelming choice.

However, legalizing PAD instead exacerbates the problem of choice at the end of life without addressing the underlying problem of overly aggressive care and insufficient guidance and access to palliative care options. Indeed, legalization of PAD in some states has only increased confusion, as hospitals and clinicians struggle themselves to understand the implications of the law. Disruptions in continuity of care for seekers of PAD are a frequent challenge when their physicians are unwilling to prescribe the lethal drug. Furthermore, legalization of PAD sends a social message that the only way to fix the structural problems in end-of-life care is to take matters into one's own hands and end one's own life.

In order to improve end-of-life care, the medical profession and organized medicine must understand the socio-cultural context from which support for PAD arose. In states where PAD has been legalized, refusal to accept this new reality and to craft an ethical response threatens to further erode the physician-patient relationship $^{12}$ and abandon patients who are seeking professional guidance and support at the end of life. This includes among others, establishing organizational priorities focused on palliative and end-of-life care, addressing clinician burnout which hinders clinicians' abilities to provide compassionate care, and re-calibrating the culture of medicine away from a consumerist mindset back towards a more humanistic profession that truly seeks to heal.

This editorial builds on ideas the author first described in a Geripal.com blog entitled "Aid in Dying: a triumph of choice over care?", published on June 7, 2016.

\section{Acknowledgements:}

The author would like to thank J. Randall Curtis, MD, MPH (University of Washington), Laura Petrillo, MD (Harvard University), and Tobias Häusermann, PhD (University of California, San Francisco) for helpful feedback on drafts of this editorial.

Funding/Support: Research reported in this publication was supported by the National Center for Advancing Translational Sciences of the NIH under Award Number KL2TR001870. Atlantic Fellowship for Equity in Brain Health, Global Brain Health Institute, UCSF. National Palliative Care Research Center Junior Investigator Career Development Award. The content is solely the responsibility of the authors and does not necessarily represent the official views of the NIH.

Corresponding Author: Elizabeth Dzeng, MD, PhD, MPH; Department of Medicine, Division of Palliative MedicineUniversity of California, San Francisco, San Francisco, CA, USA (e-mail: liz.dzeng@ucsf. edu).

\section{Compliance with Ethical Standards:}

Conflict of Interest: The author has completed and submitted the ICMJE Form for Disclosure of Potential Conflicts of Interest. There are no disclosures or conflicts of interest to report.

\section{REFERENCES}

1. Sulmasy DP, Finlay I, Fitzgerald F, Foley K, Payne R, Siegler M. PhysicianAssisted Suicide: Why Neutrality by Organized Medicine is Neither Neutral nor Appropriate. J Gen Intern Med. 2018. https://doi.org/10. 1007/s11606-018-4424-8

2. Teno JM, Gozalo PL, Bynum JPW, et al. Change in End-of-Life Care for Medicare Beneficiaries: Site of Death, Place of Care, and Health Care Transitions in 2000, 2005, and 2009. J Am Med Assoc. 2013;309(5):470477.

3. Chang D, Shapiro M. Association Between Intensive Care Unit Utilization During Hospitalization and Costs, Use of Invasive Procedures, and Mortality. JAMA Intern Med. 2016;176(10):1492-1499. https://doi.org/ 10.1001/jamainternmed.2016.4298.

4. Guidet B, Leblanc G, Simon T, et al. Mortality Among Critically Ill Elderly Patients in France. J Am Med Assoc. 2017:1-10. https://doi.org/10. 1001/jama.2017.13889.

5. Prigerson H, Bao Y, Shah M, et al. Chemotherapy Use, Performance Status, and Quality of Life at the End of Life. JAMA Oncol. 2015;1(6):778784. https://doi.org/10.1001/jamaoncol.2015.2378.

6. Wright AA, Ray A, Mack JW, et al. Associations Between End-of-Life Discussions, Patient Mental Health, Medical Care Near Death, and Caregiver Bereavement Adjustment. $J$ Am Med Assoc. 2008;300(14):1665-1673.

7. Gawande A. Letting Go: What should medicine do when it can't save your life. New Yorker. 2010. http://www.newyorker.com/reporting/2010/08/ 02/100802fa_fact_gawande. 
8. Butler K. What Broke My Father's Heart. New York Times. http://www. nytimes.com/2010/06/20/magazine/20pacemaker-t.html? pagewanted=all. Published June 16, 2010.

9. Tocqueville A de. Democracy in America. 1st ed. (Harvey M, Winthrop D, eds.). Chicago, IL: University of Chicago Press; 2002.

10. Parsons T. Illness and the Role of the Physician: A Sociological Perspective. Am J Orthopsychiatry. 1951;21(3):452-460.
11. Dzeng E, Colaianni A, Roland M, et al. Influence of Institutional Culture and Policies on Do-Not-Resuscitate Decision Making at the End of Life. JAMA Intern Med. 2015;175(5):812-819. https://doi.org/10.1001/ jamainternmed.2015.0295.

12. Seipel T. UCSF sued for refusing to help woman die. The Mercury News. https://www.mercurynews.com/2017/07/17/family-sues-ucsf-foragreeing-then-refusing-to-help-woman-die/. Published July 17, 2017. 